\author{
Waseem A. Khan, M. Ghayasuddin and M. Shadab
}

\title{
MULTIPLE-POLY-BERNOULLI POLYNOMIALS OF THE SECOND KIND ASSOCIATED WITH HERMITE POLYNOMIALS
}

\begin{abstract}
In this paper, we introduce a new class of Hermite multiple-poly-Bernoulli numbers and polynomials of the second kind and investigate some properties for these polynomials. We derive some implicit summation formulae and general symmetry identities by using different analytical means and applying generating functions. The results derived here are a generalization of some known summation formulae earlier studied by Pathan and Khan.
\end{abstract}

KEY WORDS: Hermite polynomials, multi-poly-Bernoulli polynomials of the second kind, Hermite multi-poly-Bernoulli polynomials of the second kind, summation formulae, symmetric identities.

AMS Mathematics Subject Classification: 11B68, 11B83, 33C45.

\section{Introduction}

The 2-variable Hermite Kampé de Fériet polynomials (2VHKdFP) $[3,5]$ $H_{n}(x, y)$ are defined as:

$$
H_{n}(x, y)=n ! \sum_{r=0}^{\left[\frac{n}{2}\right]} \frac{y^{r} x^{n-2 r}}{r !(n-2 r) !} .
$$

It is easily seen from definition (1) that

$$
H_{n}(2 x,-1)=H_{n}(x)
$$

and

$$
H_{n}\left(x,-\frac{1}{2}\right)=H e_{n}(x),
$$

where $H_{n}(x)$ and $H_{n}(x)$ being ordinary Hermite polynomials [1]. Also

$$
H_{n}(x, 0)=x^{n} .
$$


The generating function for Hermite polynomial $H_{n}(\mathrm{x}, \mathrm{y})$ are given by [1, 13]:

$$
e^{x t+y t^{2}}=\sum_{n=0}^{\infty} H_{n}(x, y) \frac{t^{n}}{n !}
$$

For each integers $k \geq 0$ and $n \geq 1$, the alternating sum $T_{k}(n)$ is defined by

$$
T_{k}(n)=\sum_{r=0}^{(n-1)}(-1)^{r} r^{k}=0^{k}-1^{k}+2^{k}-\cdots+(-1)^{n-1}(n-1)^{k},
$$

and the generating function is

$$
\sum_{k=0}^{\infty} T_{k}(n) \frac{t^{k}}{k !}=\frac{1-\left(-e^{t}\right)^{(n+1)}}{1+e^{t}} .
$$

In [6], Kaneko introduced and studied poly-Bernoulli numbers which generalize the classical Bernoulli numbers. Poly-Bernoulli numbers $B_{n}^{(k)}$ with $k \in \mathbb{Z}$ and $n \in \mathbb{N}$, appear the following power series:

$$
\frac{\operatorname{Li}_{k}\left(1-e^{-t}\right)}{1-e^{-t}}=\sum_{n=0}^{\infty} B_{n}^{(k)} \frac{t^{n}}{n !}
$$

where $k \in \mathbb{Z}$ and

$$
\operatorname{Li}_{k}(z)=\sum_{m=1}^{\infty} \frac{z^{m}}{m^{k}}, \quad|z|<1
$$

So for $k \leq 1$,

$$
\operatorname{Li}_{k}(z)=-\ln (1-z), \quad \operatorname{Li}_{0}(z)=\frac{z}{1-z}, \quad \operatorname{Li}_{-1}(z)=\frac{z}{(1-z)^{2}}, \ldots
$$

Moreover when $k \geq 1$, the left hand side of (4) can be written in the form

$$
e^{t} \underbrace{\frac{1}{e^{t}-1} \int_{0}^{t} \frac{1}{e^{t}-1} \cdots \int_{0}^{t} \frac{1}{e^{t}-1} \int_{0}^{t} \frac{t}{e^{t}-1}}_{(k-1) \text {-times }} d t d t \cdots d t=\sum_{n=0}^{\infty} B_{n}^{(k)} \frac{t^{n}}{n !}
$$

and multiple version of poly-Bernoulli numbers $B_{n}^{(k)}$ are defined in (see [2., p202, Remark (ii)]):

$$
\frac{\operatorname{Li}_{k_{1}, \cdots, k_{r}}\left(1-e^{-t}\right)}{\left(1-e^{-t}\right)^{r}}=\sum_{n=0}^{\infty} B_{n}^{\left(k_{1}, \cdots, k_{r}\right)} \frac{t^{n}}{n !}
$$


where

$$
\operatorname{Li}_{k_{1}, \cdots, k_{r}}(z)=\sum_{0<m_{1}<\cdots<m_{r}} \frac{z^{m_{r}}}{m_{1}^{k_{1}} \cdots m_{r} k_{r}}
$$

is the multiple polylogarithm.

Recently, Kim et al. [8] introduced the poly-Bernoulli polynomials of the second kind defined by the generating function

$$
\frac{\operatorname{Li}_{k}\left(1-e^{-t}\right)}{\log (1+t)}(1+t)^{x}=\sum_{n=0}^{\infty} b_{n}^{(k)}(x) \frac{t^{n}}{n !} .
$$

Note that

$$
\begin{aligned}
\sum_{n=0}^{\infty} b_{n}^{(1)}(x) \frac{t^{n}}{n !} & =\frac{\operatorname{Li}_{1}\left(1-e^{-t}\right)}{\log (1+t)}(1+t)^{x}=\frac{t}{\log (1+t)}(1+t)^{x} \\
& =\sum_{n=0}^{\infty} b_{n}(x) \frac{t^{n}}{n !}
\end{aligned}
$$

where $b_{n}(x)$ are called the Bernoulli numbers of the second kind (see $[4,11]$ ).

As it is well known, the higher Bernoulli polynomials of the second kind defined by the generating function (see $[4,13])$ :

$$
\left[\frac{t}{\log (1+t)}\right]^{r}(1+t)^{x}=\sum_{n=0}^{\infty} b_{n}^{(r)}(x) \frac{t^{n}}{n !},
$$

Indeed, we note that

$$
b_{n}^{(r)}(x)=B_{n}^{(n-r+1)}(x+1)
$$

where $B_{n}^{(r)}(x)$ are called the higher order Bernoulli polynomials of the first kind given by the generating function (see $[7,8,9,10]$ ):

$$
\left(\frac{t}{e^{t}-1}\right)^{r} e^{x t}=\sum_{n=0}^{\infty} B_{n}^{(r)}(x) \frac{t^{n}}{n !}
$$

Pathan and Khan [9] introduced the following generalized Hermite-Bernoulli polynomials of two variables ${ }_{H} B_{n}^{(\alpha)}(x, y)$ :

$$
\left(\frac{t}{e^{t}-1}\right)^{\alpha} e^{x t+y t^{2}}=\sum_{n=0}^{\infty} H_{n}^{(\alpha)}(x, y) \frac{t^{n}}{n !} .
$$


Setting $\alpha=1$ in (11), the result reduces to known result of Dattoli et al. [5, p.386(1.6)]:

$$
\left(\frac{t}{e^{t}-1}\right) e^{x t+y t^{2}}=\sum_{n=0}^{\infty}{ }_{H} B_{n}(x, y) \frac{t^{n}}{n !}
$$

The Stirling number of the first kind is given by

$$
(x)_{n}=x(x-1) \cdots(x-n+1)=\sum_{l=0}^{n} S_{1}(n, l) x^{l},(n \geq 0),
$$

and the Stirling number of the second kind is defined by the following generating function to be

$$
\left(e^{t}-1\right)^{n}=n ! \sum_{l=n}^{\infty} S_{2}(l, n) \frac{t^{l}}{l !}
$$

In this paper, we consider Hermite multi-poly-Bernoulli polynomials of the second kind ${ }_{H} b_{n}^{\left(k_{1}, \cdots, k_{r}\right)}(x, y, z)$ and give some formulae of these polynomials related to the Stirling numbers of the second kind. These results extend some known summations and identities of generalized Hermite multipoly-Bernoulli numbers and polynomials of the second kind studied by Qi et al. [11], Dattoli et al. [5], Khan [7] and Pathan and Khan [9, 10].

\section{Hermite multiple-poly-Bernoulli numbers and polynomials of the second kind}

In this section, we give a note on Hermite multiple-poly-Bernoulli numbers and polynomials of the second kind and investigate its properties.

Definition 1. For $k_{1}, k_{2}, \cdots, k_{r} \in \mathbb{Z}$, we define the Hermite multiple-polyBernoulli polynomials of the second kind ${ }_{H} b_{n}^{\left(k_{1}, k_{2}, \cdots, k_{r}\right)}(x, y, z)$ by the generating function:

$$
\frac{r ! \operatorname{Li}_{k_{1}, k_{2}, \cdots, k_{r}}\left(1-e^{-t}\right)}{[\log (1+t)]^{r}}(1+t)^{x} e^{y t+z t^{2}}=\sum_{n=0}^{\infty} H b_{n}^{\left(k_{1}, k_{2}, \cdots, k_{r}\right)}(x, y, z) \frac{t^{n}}{n !},
$$

so that

$$
{ }_{H} b_{n}^{\left(k_{1}, k_{2}, \cdots, k_{r}\right)}(x, y, z)=\sum_{m=0}^{n}\left(\begin{array}{c}
n \\
m
\end{array}\right) b_{n-m}^{\left(k_{1}, k_{2}, \cdots, k_{r}\right)} H_{m}(y, z) .
$$


When $x=y=z=0, b_{n}^{\left(k_{1}, k_{2}, \cdots, k_{r}\right)}={ }_{H} b_{n}^{\left(k_{1}, k_{2}, \cdots, k_{r}\right)}(0,0,0)$ are called the multi-poly-Bernoulli numbers of the second kind. From (15), we note that

$$
\begin{aligned}
\sum_{n=0}^{\infty}{ }_{H} b_{n}^{(1,1, \cdots, 1)}(x, y) \frac{t^{n}}{n !} & =\frac{r ! \operatorname{Li}_{1,1, \cdots, 1}\left(1-e^{-t}\right)}{[\log (1+t)]^{r}}(1+t)^{x} e^{y t+z t^{2}} \\
& =\sum_{n=0}^{\infty} H_{n}^{(r)}(x, y, z) \frac{t^{n}}{n !} .
\end{aligned}
$$

Thus, we have

$$
{ }_{H} b_{n}^{(1,1, \cdots, 1)}(x, y, z)={ }_{H} b_{n}^{(r)}(x, y, z), \quad n \geq 0 .
$$

Remark 1. On setting $y=z=0$, (15) reduces to the following multi-poly-Bernoulli polynomials of the second kind defined by Qi et al. [11, p.3 Eq.(2.1)]:

$$
\frac{r ! \mathrm{Li}_{k_{1}, k_{2}, \cdots, k_{r}}\left(1-e^{-t}\right)}{[\log (1+t)]^{r}}(1+t)^{x}=\sum_{n=0}^{\infty} b_{n}^{\left(k_{1}, k_{2}, \cdots, k_{r}\right)}(x) \frac{t^{n}}{n !},
$$

$k_{1}, k_{2}, \cdots, k_{r} \in \mathbb{Z}$.

Theorem 1. For $n \geq 0$, we have

$$
{ }_{H} b_{n}^{(2)}(x, y, z)=\sum_{m=0}^{n}\left(\begin{array}{l}
n \\
m
\end{array}\right) \frac{B_{m} m !}{m+1} H b_{n-m}(x, y, z) .
$$

Proof. From definition (15), we have

$$
\begin{aligned}
& \sum_{n=0}^{\infty} H_{n} b_{n}^{\left(k_{1}, k_{2}, \cdots, k_{r}\right)}(x, y, z) \frac{t^{n}}{n !}=\frac{r ! \operatorname{Li}_{k_{1}}, k_{2}, \cdots, k_{r}\left(1-e^{-t}\right)}{[\log (1+t)]^{r}}(1+t)^{x} e^{y t+z t^{2}} \\
& =\frac{r !(1+t)^{x} e^{y t+z t^{2}}}{[\log (1+t)]^{r}} \operatorname{Li}_{k_{1}, k_{2}, \cdots, k_{r}}\left(1-e^{-t}\right) \\
& =\frac{r !(1+t)^{x} e^{y t+z t^{2}}}{[\log (1+t)]^{r}} \int_{0}^{t} \int_{0}^{t_{b_{1}}+\cdots+b_{h}} \cdots \underbrace{\int_{0}^{t_{2}} \frac{1}{e^{t_{b_{1}}+\cdots+b_{h}}-1} \cdots \frac{1}{e^{t_{b_{1}+\cdots+b_{h-1}+2}}-1}}_{\left(b_{h}-1\right)-\text { times }} \\
& \times \frac{1}{a_{h} !} \frac{\left(t_{b_{1}+\cdots+b_{h-1}+1}-t_{b_{1}+\cdots+b_{h-1}}\right)^{a_{h}}}{e^{t_{1}+\cdots+b_{h-1}+1}-1} \cdot \underbrace{\frac{1}{e^{t_{b_{1}+\cdots+b_{h-1}+1}}-1} \cdots \frac{1}{e^{t_{b_{1}+\cdots+b_{h-2}+2}-1}}}_{\left(b_{h-1}-1\right)-\text { times }} \\
& \times \cdots \cdots \times \frac{1}{a_{3} !} \frac{\left(t_{b_{1}+b_{2}+1}-t_{b_{1}+b_{2}}\right)^{a_{3}}}{e^{t_{b_{1}+b_{2}+1}}-1} \cdot \underbrace{\frac{1}{e^{t_{b_{1}+b_{2}}}-1} \cdots \frac{1}{e^{t_{b_{1}+2}}-1}}_{\left(b_{2}-1\right)-\text { times }} \\
& \times \frac{1}{a_{2} !} \frac{\left(t_{b_{1}+1}-t_{b_{1}}\right)^{a_{2}}}{e^{t_{b_{1}+1}}-1} \cdot \underbrace{\frac{1}{e^{t_{b_{1}}}-1} \cdots \frac{1}{e^{t_{2}}-1}}_{\left(b_{1}-1\right)-\text { times }} \cdot \frac{1}{a_{1} !} \frac{t_{1}^{a_{1}}}{e^{t_{1}}-1} d t_{1} d t_{2} \cdots d t_{b_{1}+\cdots+b_{h}} .
\end{aligned}
$$


For $r=1$

$$
\begin{aligned}
& \sum_{n=0}^{\infty} H b_{n}^{\left(k_{1}\right)}(x, y, z) \frac{t^{n}}{n !} \\
& =\frac{(1+t)^{x} e^{y t+z t^{2}}}{\log (1+t)} \underbrace{\int_{0}^{t} \frac{1}{e^{z}-1} \int_{0}^{t} \frac{1}{e^{z}-1} \cdots \frac{1}{e^{z}-1} \int_{0}^{t} \frac{z}{e^{z}-1}}_{\left(k_{1}-1\right)-\text { times }} d z \cdots d z .
\end{aligned}
$$

In particular $k_{1}=2$, we have

$$
\begin{aligned}
\sum_{n=0}^{\infty}{ }_{H} b_{n}^{(2)}(x, y, z) \frac{t^{n}}{n !} & =\frac{(1+t)^{x} e^{y t+z t^{2}}}{\log (1+t)} \int_{0}^{t} \frac{z}{e^{z}-1} d z \\
& =\left(\sum_{m=0}^{\infty} \frac{t^{m} B_{m} m !}{m+1 m !}\right) \frac{t}{\log (1+t)}(1+t)^{x} e^{y t+z t^{2}} \\
& =\left(\sum_{m=0}^{\infty} \frac{t^{m} B_{m} m !}{m+1 m !}\right)\left(\sum_{n=0}^{\infty} H b_{n}(x, y, z) \frac{t^{n}}{n !}\right)
\end{aligned}
$$

Replacing $n$ by $n-m$ in the r.h.s. of above equation, we have

$$
\sum_{n=0}^{\infty} H_{n}^{(2)}(x, y, z) \frac{t^{n}}{n !}=\sum_{n=0}^{\infty} \sum_{m=0}^{n}\left(\begin{array}{c}
n \\
m
\end{array}\right) \frac{B_{m} m !}{m+1} H_{n-m}(x, y, z) \frac{t^{n}}{n !} .
$$

On equating the coefficients of the like powers of $\frac{t^{n}}{n !}$ in the above equation, we get the result $(20)$.

Theorem 2. For $n \geq 1$, the degree of ${ }_{H} b_{n}^{\left(k_{1}, k_{2}, \cdots, k_{r}\right)}(x, y, z)$ is $n-1$, we have

$$
\frac{H_{n}^{\left(k_{1}, k_{2}, \cdots, k_{r}\right)}(x, y, z)}{n}=\sum_{m=0}^{n-1}\left(\begin{array}{l}
n-1 \\
m
\end{array}\right) \frac{b_{m+1}^{\left(k_{1}, k_{2}, \cdots, k_{r}\right)}(x)}{m+1} H_{n-m-1}(y, z)
$$

Proof. By Definition 1 of Hermite multi-poly-Bernoulli polynomials of the second kind, we have

$$
\begin{aligned}
\sum_{n=0}^{\infty} H_{n} b_{n}^{\left(k_{1}, k_{2}, \cdots, k_{r}\right)}(x, y, z) \frac{t^{n}}{n !} & =\frac{r ! \operatorname{Li}_{k_{1}, k_{2}, \cdots, k_{r}}\left(1-e^{-t}\right)}{[\log (1+t)]^{r}}(1+t)^{x} e^{y t+z t^{2}} \\
& =\left(\sum_{m=0}^{\infty} b_{m}^{\left(k_{1}, k_{2}, \cdots, k_{r}\right)}(x) \frac{t^{m}}{m !}\right)\left(\sum_{n=0}^{\infty} H_{n}(y, z) \frac{t^{n}}{n !}\right) .
\end{aligned}
$$


Replacing $n$ by $n-m$ in the r.h.s. of above equation and comparing the coefficients of $\frac{t^{n}}{n !}$, we get

$$
H_{n}^{\left(k_{1}, k_{2}, \cdots, k_{r}\right)}(x, y, z)=\sum_{m=0}^{n}\left(\begin{array}{l}
n \\
m
\end{array}\right) b_{m}^{\left(k_{1}, k_{2}, \cdots, k_{r}\right)}(x) H_{n-m}(y, z),
$$

$n \geq 0$. From (22), we have

$$
\frac{H_{n} b_{n}^{\left(k_{1}, k_{2}, \cdots, k_{r}\right)}(x, y, z)}{n}=\sum_{m=0}^{n-1}\left(\begin{array}{l}
n-1 \\
m
\end{array}\right) \frac{b_{m+1}^{\left(k_{1}, k_{2}, \cdots, k_{r}\right)}(x)}{m+1} H_{n-m-1}(y, z)
$$

$n \geq 1$. Therefore by (23), we get the result (21).

Theorem 3. For $n \geq 0$, and $r \in \mathbb{N}$ with $n \geq r$, we have

$$
\begin{aligned}
H & b_{n-r}^{\left(k_{1}, k_{2}, \cdots, k_{r}\right)}(x, y, z)\left(\begin{array}{c}
n \\
r
\end{array}\right) \\
= & \sum_{l=0}^{n} \sum_{m_{r}=0}^{n-l} \sum_{0<m_{1}<m_{2}<\cdots<m_{r}}\left(\begin{array}{c}
n \\
l
\end{array}\right) H_{l}^{(r)}(x, y, z) \\
& \times \frac{(-1)^{m_{r}-n-l} m_{r} ! S_{2}\left(n-l, m_{r}\right)}{m_{1}^{k_{1}} m_{2}^{k_{2}} \cdots m_{r}^{k_{r}}} .
\end{aligned}
$$

Proof. From equation (15), we have

$$
\begin{aligned}
& \sum_{n=0}^{\infty} H b_{n}^{\left(k_{1}, k_{2}, \cdots, k_{r}\right)}(x, y, z) \frac{t^{n}}{n !}=\frac{r !(1+t)^{x}\left(e^{y t+z t^{2}}\right.}{[\log (1+t)]^{r}} \operatorname{Li}_{k_{1}, k_{2}, \cdots, k_{r}}\left(1-e^{-t}\right) \\
& =\left(\frac{r !(1+t)^{x} e^{y t+z t^{2}}}{[\log (1+t)]^{r}}\right) \sum_{0<m_{1}<m_{2}<\cdots<m_{r}} \frac{\left(1-e^{-t}\right)^{m_{r}}}{m_{1}^{k_{1}} m_{2}^{k_{2}} \cdots m_{r}^{k_{r}}} \\
& =\left(\frac{r !(1+t)^{x} e^{y t+z t^{2}}}{[\log (1+t)]^{r}}\right) \sum_{0<m_{1}<m_{2}<\cdots<m_{r}} \frac{(-1)^{m_{r}} m_{r} !}{m_{1}^{k_{1}} m_{2}^{k_{2}} \cdots m_{r}^{k_{r}}} \\
& \quad \times \sum_{n=m_{r}}^{\infty}(-1)^{n} S_{2}\left(n, m_{r}\right) \frac{t^{n}}{n !} \\
& =\left(\frac{r !(1+t)^{x} e^{y t+z t^{2}}}{[\log (1+t)]^{r}}\right) \\
& \quad \times \sum_{n=0}^{\infty}\left[\sum_{m_{r}=0}^{n} \sum_{0<m_{1}<m_{2}<\cdots<m_{r}} \frac{(-1)^{m_{r}} m_{r} !(-1)^{n} S_{2}\left(n, m_{r}\right)}{m_{1}^{k_{1}} m_{2}^{k_{2}} \cdots m_{r}^{k_{r}}}\right] \frac{t^{n}}{n !}
\end{aligned}
$$




$$
\begin{aligned}
& =\frac{r !}{t^{r}}\left(\frac{t}{\log (1+t)}\right)^{r}(1+t)^{x} e^{y t+z t^{2}} \\
& \times \sum_{n=0}^{\infty}\left[\sum_{m_{r}=0}^{n} \sum_{0<m_{1}<m_{2}<\cdots<m_{r}} \frac{(-1)^{m_{r}} m_{r} !(-1)^{n} S_{2}\left(n, m_{r}\right)}{m_{1}^{k_{1}} m_{2}^{k_{2}} \cdots m_{r}^{k_{r}}}\right] \frac{t^{n}}{n !} \\
& \times \sum_{n=0}^{\infty} H b_{n}^{\left(k_{1}, k_{2}, \cdots, k_{r}\right)}(x, y, z) \frac{t^{n+r}}{n ! r !} \\
& =\sum_{l=0}^{\infty} H_{l}^{(r)}(x, y, z) \frac{t^{l}}{l !} \\
& \times \sum_{n=0}^{\infty}\left[\sum_{m_{r}=0}^{n} \sum_{0<m_{1}<m_{2}<\cdots<m_{r}} \frac{(-1)^{m_{r}} m_{r} !(-1)^{n} S_{2}\left(n, m_{r}\right)}{m_{1}^{k_{1}} m_{2}^{k_{2}} \cdots m_{r}^{k_{r}}}\right] \frac{t^{n}}{n !} \\
& \times \sum_{n=0}^{\infty} H b_{n}^{\left(k_{1}, k_{2}, \cdots, k_{r}\right)}(x, y, z) \frac{t^{n+r}}{n ! r !} \\
& =\sum_{n=0}^{\infty}\left[\sum_{l=0}^{n} \sum_{m_{r}=0}^{n-l} \sum_{0<m_{1}<m_{2}<\cdots<m_{r}}\left(\begin{array}{c}
n \\
l
\end{array}\right) H_{l} b_{l}^{(r)}(x, y, z)\right. \\
& \left.\times \frac{(-1)^{m_{r}-n-l} m_{r} ! S_{2}\left(n-l, m_{r}\right)}{m_{1}^{k_{1}} m_{2}^{k_{2}} \cdots m_{r}^{k_{r}}}\right] \frac{t^{n}}{n !} \\
& \times \sum_{n=r}^{\infty} H b_{n-r}^{\left(k_{1}, k_{2}, \cdots, k_{r}\right)}(x, y)\left(\begin{array}{c}
n \\
r
\end{array}\right) \frac{t^{n}}{n !} \\
& =\sum_{n=0}^{\infty}\left[\sum_{l=0}^{n} \sum_{m_{r}=0}^{n-l} \sum_{0<m_{1}<m_{2}<\cdots<m_{r}}\left(\begin{array}{c}
n \\
l
\end{array}\right){ }_{H} b_{l}^{(r)}(x, y, z)\right. \\
& \left.\times \frac{(-1)^{m_{r}-n-l} m_{r} ! S_{2}\left(n-l, m_{r}\right)}{m_{1}^{k_{1}} m_{2}^{k_{2}} \cdots m_{r}^{k_{r}}}\right] \frac{t^{n}}{n !} .
\end{aligned}
$$

Comparing the coefficients of $\frac{t^{n}}{n !}$, we arrive at the desired result (24).

Remark 2. For $y=z=0$, Theorem 3 reduces to the known result of Qi et al. [11.,p. 5, Theorem (2.3)].

Corollary 1. For $n \geq 0$, and $r \in \mathbb{N}$ with $n \geq r$, we have

$$
\begin{aligned}
(26) b_{n-r}^{\left(k_{1}, k_{2}, \cdots, k_{r}\right)}(x)\left(\begin{array}{c}
n \\
r
\end{array}\right)= & \sum_{l=0}^{n} \sum_{m_{r}=0}^{n-l} \sum_{0<m_{1}<m_{2}<\cdots<m_{r}}\left(\begin{array}{c}
n \\
l
\end{array}\right) b_{l}^{(r)}(x) \\
& \times \frac{(-1)^{m_{r}-n-l} m_{r} ! S_{2}\left(n-l, m_{r}\right)}{m_{1}^{k_{1}} m_{2}^{k_{2}} \cdots m_{r}^{k_{r}}}
\end{aligned}
$$


Theorem 4. For $n \geq 0$, we have

$$
\begin{gathered}
H b_{n}^{\left(k_{1}, k_{2}, \cdots, k_{r}\right)}(x+1, y, z)-{ }_{H} b_{n}^{\left(k_{1}, k_{2}, \cdots, k_{r}\right)}(x, y, z) \\
=n_{H} b_{n-1}^{\left(k_{1}, k_{2}, \cdots, k_{r}\right)}(x, y, z)
\end{gathered}
$$

Proof. Using Definition 1, we have

$$
\begin{aligned}
\sum_{n=0}^{\infty} H_{n} & b_{n}^{\left(k_{1}, k_{2}, \cdots, k_{r}\right)}(x+1, y, z) \frac{t^{n}}{n !}-\sum_{n=0}^{\infty}{ }_{H} b_{n}^{\left(k_{1}, k_{2}, \cdots, k_{r}\right)}(x, y, z) \frac{t^{n}}{n !} \\
= & \frac{r ! \operatorname{Li}_{k_{1}, k_{2}, \cdots, k_{r}}\left(1-e^{-t}\right)}{[\log (1+t)]^{r}}(1+t)^{x+1} e^{y t+z t^{2}} \\
& -\frac{r ! \operatorname{Li}_{k_{1}, k_{2}, \cdots, k_{r}}\left(1-e^{-t}\right)}{[\log (1+t)]^{r}}(1+t)^{x} e^{y t+z t^{2}} \\
= & \frac{r ! \operatorname{Li}_{k_{1}, k_{2}, \cdots, k_{r}}\left(1-e^{-t}\right) t}{[\log (1+t)]^{r}}(1+t)^{x} e^{y t+z t^{2}} \\
= & \sum_{n=1}^{\infty} n_{H} b_{n-1}^{\left(k_{1}, k_{2}, \cdots, k_{r}\right)}(x, y, z) \frac{t^{n}}{n !} .
\end{aligned}
$$

Comparing the coefficients of $\frac{t^{n}}{n !}$, we get the result (27).

Remark 3. For $y=z=0$, Theorem 4 reduces to the known result of Qi et al. [11.,p. 3, Theorem (2.1)].

Corollary 2. For $n \geq 0$, we have

$$
b_{n}^{\left(k_{1}, k_{2}, \cdots, k_{r}\right)}(x+1)-b_{n}^{\left(k_{1}, k_{2}, \cdots, k_{r}\right)}(x)=n b_{n-1}^{\left(k_{1}, k_{2}, \cdots, k_{r}\right)}(x) .
$$

\section{Implicit formulae involving Hermite multiple-poly- Bernoulli polynomials of the second kind}

In this section, we derive some implicit summation formulae for Hermite multiple-poly-Bernoulli polynomials of the second kind $H_{n}^{\left(k_{1}, k_{2}, \cdots, k_{r}\right)}(x, y)$. We now begin with the following theorems.

Theorem 5. The following implicit summation formulae for multiple Hermite poly-Bernoulli polynomials ${ }_{H} b_{n}^{\left(k_{1}, k_{2}, \cdots, k_{r}\right)}(x, y, z)$ holds true:

$$
\begin{aligned}
H_{l+p} b_{l+p}^{\left(k_{1}, k_{2}, \cdots, k_{r}\right)}(x, w, z) & \\
& =\sum_{m, n=0}^{l, p}\left(\begin{array}{l}
l \\
m
\end{array}\right)\left(\begin{array}{l}
p \\
n
\end{array}\right)(w-y)^{m+n} H_{l+p-m-n}^{\left(k_{1}, k_{2}, \cdots, k_{r}\right)}(x, y, z) .
\end{aligned}
$$


Proof. We replace t by $t+u$ and rewrite the generating function (15) as

$$
\begin{aligned}
& \frac{\operatorname{Li}_{k_{1}, k_{2}, \cdots, k_{r}}\left(1-e^{-(t+u)}\right)}{e^{t+u}-1} e^{z(t+u)^{2}}\left(1+(t+u)^{x}\right) \\
& =e^{-y(t+u)} \sum_{l, p=0}^{\infty} H b_{l+p}^{\left(k_{1}, k_{2}, \cdots, k_{r}\right)}(x, y, z) \frac{t^{l}}{l !} \frac{u^{p}}{p !} .
\end{aligned}
$$

Replacing $y$ by $w$ in the above equation and equating the resulting equation to the above equation, we get

$$
\begin{aligned}
e^{(w-y)(t+u)} & \sum_{m, l=0}^{\infty} H b_{l+p}^{\left(k_{1}, k_{2}, \cdots, k_{r}\right)}(x, y, z) \frac{t^{l}}{l !} \frac{u^{p}}{p !} \\
= & \sum_{l, p=0}^{\infty} H b_{l+p}^{\left(k_{1}, k_{2}, \cdots, k_{r}\right)}(x, w, z) \frac{t^{l}}{l !} \frac{u^{p}}{p !} .
\end{aligned}
$$

On expanding exponential function, (31) gives

$$
\begin{gathered}
\sum_{N=0}^{\infty} \frac{[(w-y)(t+u)]^{N}}{N !} \sum_{l, p=0}^{\infty} H_{l+p}^{\left(k_{1}, k_{2}, \cdots, k_{r}\right)}(x, y, z) \frac{t^{l}}{l !} \frac{u^{p}}{p !} \\
=\sum_{l, p=0}^{\infty} H b_{l+p}^{\left(k_{1}, k_{2}, \cdots, k_{r}\right)}(x, w, z) \frac{t^{l}}{l !} \frac{u^{p}}{p !}
\end{gathered}
$$

which on using formula [13, p.52(2)]:

$$
\sum_{N=0}^{\infty} f(N) \frac{(x+y)^{N}}{N !}=\sum_{n, m=0}^{\infty} f(n+m) \frac{x^{n}}{n !} \frac{y^{m}}{m !},
$$

in the left hand side of (32), we get

$$
\begin{gathered}
\sum_{m, n=0}^{\infty} \frac{(w-y)^{m+n} t^{m} u^{n}}{m ! n !} \sum_{l, p=0}^{\infty} H b_{l+p}^{\left(k_{1}, k_{2}, \cdots, k_{r}\right)}(x, y, z) \frac{t^{l}}{l !} \frac{u^{p}}{p !} \\
=\sum_{l, p=0}^{\infty} H_{l+p}^{\left(k_{1}, k_{2}, \cdots, k_{r}\right)}(x, w, z) \frac{t^{l}}{l !} \frac{u^{p}}{p !} .
\end{gathered}
$$

Now replacing $l$ by $l-m, p$ by $p-n$ and using the lemma [13, p.100(1)] in the left hand side of (34), we get

$$
\begin{gathered}
\sum_{m, n=0}^{\infty} \sum_{l, p=0}^{\infty} \frac{(w-y)^{m+n}}{m ! n !} H_{l+p-m-n}^{\left(k_{1}, k_{2}, \cdots, k_{r}\right)}(x, y, z) \frac{t^{l}}{(l-m) !} \frac{u^{p}}{(p-n) !} \\
=\sum_{l, p=0}^{\infty} H b_{l+p}^{\left(k_{1}, k_{2}, \cdots, k_{r}\right)}(x, w, z) \frac{t^{l}}{l !} \frac{u^{p}}{p !}
\end{gathered}
$$


Finally, on equating the coefficients of the like powers of $t$ and $u$ in the above equation, we get the required result.

Remark 4. By taking $l=0$ in (29), we get the following result.

$$
{ }_{H} b_{p}^{\left(k_{1}, k_{2}, \cdots, k_{r}\right)}(x, w, z)=\sum_{n=0}^{p}\left(\begin{array}{c}
p \\
n
\end{array}\right)(w-y)^{n}{ }_{H} b_{p-n}^{\left(k_{1}, k_{2}, \cdots, k_{r}\right)}(x, y, z) .
$$

Theorem 6. For $x, y, z \in \mathbb{R}$ and $n \geq 0$, we have

$$
\begin{aligned}
& H_{n}^{\left(k_{1}, k_{2}, \cdots, k_{r}\right)}(x+u, y, z) \\
& \quad=\sum_{j=0}^{n}\left(\begin{array}{c}
n \\
j
\end{array}\right)(-1)^{j}(-u)_{j H} b_{n-j}^{\left(k_{1}, k_{2}, \cdots, k_{r}\right)}(x, y, z) .
\end{aligned}
$$

Proof. Since

$$
\begin{aligned}
\sum_{n=0}^{\infty} H b_{n}^{\left(k_{1}, k_{2}, \cdots, k_{r}\right)}(x+u, y, z) \frac{t^{n}}{n !} & \\
= & \frac{r ! \operatorname{Li}_{k_{1}, k_{2}, \cdots, k_{r}}\left(1-(e)^{-t}\right)}{[\log (1+t)]^{r}}(1+t)^{(x+u)} e^{y t+z t^{2}} . \\
& =\left(\sum_{n=0}^{\infty} H b_{n}^{\left(k_{1}, k_{2}, \cdots, k_{r}\right)}(x, y, z) \frac{t^{n}}{n !}\right)\left(\sum_{j=0}^{\infty}(-1)^{j}(-u)_{j} \frac{t^{j}}{j !}\right) .
\end{aligned}
$$

Replacing $n$ by $n-j$ in the r.h.s. of above equation, we get

$$
\begin{aligned}
\sum_{n=0}^{\infty} H b_{n}^{\left(k_{1}, k_{2}, \cdots, k_{r}\right)}(x+u, y, z) \frac{t^{n}}{n !} \\
=\sum_{n=0}^{\infty} \sum_{j=0}^{n}\left(\begin{array}{c}
n \\
j
\end{array}\right)(-1)^{j}(-u)_{j H} b_{n-j}^{\left(k_{1}, k_{2}, \cdots, k_{r}\right)}(x, y, z) \frac{t^{n}}{n !} .
\end{aligned}
$$

Comparing the coefficients of $\frac{t^{n}}{n !}$, we obtain (37).

Remark 5. On setting $u=1$ in the above theorem to get

$$
{ }_{H} b_{n}^{\left(k_{1}, k_{2}, \cdots, k_{r}\right)}(x+1, y, z)=\sum_{j=0}^{n}\left(\begin{array}{c}
n \\
j
\end{array}\right)(-1)^{j}{ }_{H} b_{n-j}^{\left(k_{1}, k_{2}, \cdots, k_{r}\right)}(x, y, z) .
$$

Theorem 7. For $x, y, z \in \mathbb{R}$ and $n \geq 0$, we have

$$
\begin{aligned}
& H b_{n}^{\left(k_{1}, k_{2}, \cdots, k_{r}\right)}(x, y+u, z+w) \\
&=\sum_{m=0}^{n}\left(\begin{array}{c}
n \\
m
\end{array}\right) H b_{n-m}^{\left(k_{1}, k_{2}, \cdots, k_{r}\right)}(x, y, z) H_{m}(u, w) .
\end{aligned}
$$


Proof. By the definition of Hermite multiple-poly-Bernoulli polynomials of the second kind and then by using (2), we have

$$
\begin{aligned}
\sum_{n=0}^{\infty} H b_{n}^{\left(k_{1}, k_{2}, \cdots, k_{r}\right)}(x, y+u, z+w) \frac{t^{n}}{n !} & \\
= & \frac{r ! \operatorname{Li}_{k_{1}, k_{2}, \cdots, k_{r}}\left(1-e^{-t}\right)}{[\log (1+t)]^{r}}(1+t)^{x} e^{(y+u) t+(z+w) t^{2}} \\
= & \left(\sum_{n=0}^{\infty} H b_{n}^{\left(k_{1}, k_{2}, \cdots, k_{r}\right)}(x, y, z) \frac{t^{n}}{n !}\right)\left(\sum_{m=0}^{\infty} H_{m}(u, w) \frac{t^{m}}{m !}\right) .
\end{aligned}
$$

Now replacing $n$ by $n-m$ in the r.h.s. of above equation and comparing the coefficients of $\frac{t^{n}}{n !}$, we get the required result (39).

Theorem 8. For $x, y, z \in \mathbb{R}$ and $n \geq 0$, we have

$$
\begin{aligned}
H b_{n}^{\left(k_{1}, k_{2}, \cdots, k_{r}\right)}(x, y, z) & \\
= & \sum_{m=0}^{n-2 j} \sum_{j=0}^{\left[\frac{n}{2}\right]} z^{j} y^{n-m-2 j} b_{m}^{\left(k_{1}, k_{2}, \cdots, k_{r}\right)}(x) \frac{n !}{m ! j !(n-2 j-m) !} .
\end{aligned}
$$

Proof. Applying the Definition 1 to the term $\frac{r ! \mathrm{Li}_{k_{1}, k_{2}, \cdots, k_{r}}\left(1-e^{-t}\right)}{[\log (1+t)]^{r}}$ and expanding the exponential function $e^{y t+z t^{2}}$ at $t=0$ yields

$$
\begin{aligned}
\sum_{n=0}^{\infty}{ }_{H} b_{n}^{\left(k_{1}, k_{2}, \cdots, k_{r}\right)}(x, y, z) \frac{t^{n}}{n !}=\frac{r ! \operatorname{Li}_{k_{1}, k_{2}, \cdots, k_{r}}\left(1-e^{-t}\right)}{[\log (1+t)]^{r}}(1+t)^{x} e^{x t+y t^{2}} \\
=\left(\sum_{m=0}^{\infty} b_{m}^{\left(k_{1}, k_{2}, \cdots, k_{r}\right)}(x) \frac{t^{m}}{m !}\right)\left(\sum_{n=0}^{\infty} y^{n} \frac{t^{n}}{n !}\right)\left(\sum_{j=0}^{\infty} z^{j} \frac{t^{2 j}}{j !}\right) \\
=\sum_{n=0}^{\infty}\left(\sum_{m=0}^{n}\left(\begin{array}{c}
n \\
m
\end{array}\right) b_{m}^{\left(k_{1}, k_{2}, \cdots, k_{r}\right)}(x) y^{n-m}\right) \frac{t^{n}}{n !}\left(\sum_{j=0}^{\infty} z^{j} \frac{t^{2 j}}{j !}\right) .
\end{aligned}
$$

Replacing $n$ by $n-2 j$ in the r.h.s. of above equation, we have

$$
\begin{aligned}
& \sum_{n=0}^{\infty} H_{n}^{\left(k_{1}, k_{2}, \cdots, k_{r}\right)}(x, y, z) \frac{t^{n}}{n !} \\
& =\sum_{n=0}^{\infty}\left(\sum_{m=0}^{n-2 j} \sum_{j=0}^{\left[\frac{n}{2}\right]}\left(\begin{array}{l}
n-2 j \\
m
\end{array}\right) b_{m}^{\left(k_{1}, k_{2}, \cdots, k_{r}\right)}(x) y^{n-m-2 j} z^{j}\right) \frac{t^{n}}{(n-2 j) ! j !} .
\end{aligned}
$$

On equating the coefficients of $\frac{t^{n}}{n !}$, we arrive at the required result (40). 
Theorem 9. For $x, y, z \in \mathbb{R}$ and $n \geq 0$, we have

$$
\begin{aligned}
H & b_{n}^{\left(k_{1}, k_{2}, \cdots, k_{r}\right)}(x, y, z) \\
& =\sum_{r=0}^{n}\left(\begin{array}{c}
n \\
r
\end{array}\right)(-1)^{r}(-w)_{r H} b_{n-r}^{\left(k_{1}, k_{2}, \cdots, k_{r}\right)}(x-w, y, z) .
\end{aligned}
$$

Proof. By exploiting the generating function (15), we have

$$
\begin{aligned}
& \frac{r ! \operatorname{Li}_{k_{1}, k_{2}, \cdots, k_{r}}\left(1-e^{-t}\right)}{[\log (1+t)]^{r}}(1+t)^{x-w+w} e^{y t+z t^{2}} \\
& \quad=\left(\sum_{n=0}^{\infty} H_{n}^{\left(k_{1}, k_{2}, \cdots, k_{r}\right)}(x-w, y, z) \frac{t^{n}}{n !}\right)\left(\sum_{r=0}^{\infty}(-1)^{r}(-w)_{r} \frac{t^{r}}{r}\right) .
\end{aligned}
$$

Replacing $n$ by $n-r$ in the r.h.s. of above equation and comparing the coefficients of $\frac{t^{n}}{n !}$, we get the result (41).

\section{Identities for Hermite multiple-poly-Bernoulli polynomials of the second kind}

In this section, we give general symmetry identity for the multiple-polyBernoulli polynomials of the second kind $b_{n}^{\left(k_{1}, k_{2}, \cdots, k_{r}\right)}(x)$ and the Hermite multiple-poly-Bernoulli polynomials of the second kind ${ }_{H} b_{n}^{\left(k_{1}, k_{2}, \cdots, k_{r}\right)}(x, y)$ by applying the generating function (15) and (18). The results extend some known identities of Khan [7], Pathan and Khan [9, 10].

Theorem 10. Let $c, d>0, c \neq d, x, y \in \mathbb{R}$ and $n \geq 0$, the following identity holds true:

$\begin{aligned} \text { (42) } & \sum_{m=0}^{n}\left(\begin{array}{l}n \\ m\end{array}\right) d^{m} c^{n-m} b_{n-m}^{\left(k_{1}, k_{2}, \cdots, k_{r}\right)}\left(d x, d y, d^{2} y\right)_{H} b_{m}^{(k)}\left(c x, c y, c^{2} y\right) \\ = & \sum_{m=0}^{n}\left(\begin{array}{l}n \\ m\end{array}\right) c^{m} d^{n-m} b_{n-m}^{\left(k_{1}, k_{2}, \cdots, k_{r}\right)}\left(c x, c y, c^{2} y\right)_{H} b_{m}^{\left(k_{1}, k_{2}, \cdots, k_{r}\right)}\left(d x, d y, d^{2} y\right) .\end{aligned}$

Proof. Start with

$$
\begin{aligned}
g(t)= & \left(\frac{\left(r ! \operatorname{Li}_{k_{1}, k_{2}, \cdots, k_{r}}\left(1-e^{-c t}\right)\right)\left(r ! \operatorname{Li}_{k_{1}, k_{2}, \cdots, k_{r}}\left(1-e^{-d t}\right)\right)}{[(\log (1+c t))]^{r}[(\log (1+d t))]^{r}}\right) \\
& \times(1+c d t)^{x} e^{c d y t+c^{2} d^{2} z t^{2}}
\end{aligned}
$$


Then the expression for $g(t)$ is symmetric in $c$ and $d$ and we can expand $g(t)$ into series in two ways to obtain

$$
\begin{gathered}
g(t)=\sum_{n=0}^{\infty} H_{n}^{\left(k_{1}, k_{2}, \cdots, k_{r}\right)}\left(d x, d y, d^{2} z\right) \frac{(c t)^{n}}{n !} \sum_{m=0}^{\infty}{ }_{H} b_{m}^{\left(k_{1}, k_{2}, \cdots, k_{r}\right)}\left(c x, c y, c^{2} y\right) \frac{(d t)^{m}}{m !} \\
=\sum_{n=0}^{\infty}\left(\sum_{m=0}^{n}\left(\begin{array}{c}
n \\
m
\end{array}\right) c^{n-m} d^{m}{ }_{H} b_{n-m}^{\left(k_{1}, k_{2}, \cdots, k_{r}\right)}\right. \\
\left.\quad \times\left(d x, d y, d^{2} z\right)_{H} b_{m}^{\left(k_{1}, k_{2}, \cdots, k_{r}\right)}\left(c x, c y, c^{2} y\right)\right) \frac{t^{n}}{n !} .
\end{gathered}
$$

Similarly, we can show that

$$
\begin{aligned}
& g(t)= \sum_{n=0}^{\infty} H b_{n}^{\left(k_{1}, k_{2}, \cdots, k_{r}\right)}\left(c x, c y, c^{2} y\right) \frac{(b t)^{n}}{n !} \sum_{m=0}^{\infty} H b_{m}^{\left(k_{1}, k_{2}, \cdots, k_{r}\right)}\left(d x, d y, d^{2} z\right) \frac{(a t)^{m}}{m !} \\
&=\sum_{n=0}^{\infty}\left(\sum_{m=0}^{n}\left(\begin{array}{c}
n \\
m
\end{array}\right) c^{m} d^{n-m}{ }_{H} b_{n-m}^{\left(k_{1}, k_{2}, \cdots, k_{r}\right)}\right. \\
&\left.\quad \times\left(c x, c y, c^{2} y\right)_{H} b_{m}^{\left(k_{1}, k_{2}, \cdots, k_{r}\right)}\left(d x, d y, d^{2} z\right)\right) \frac{t^{n}}{n !} .
\end{aligned}
$$

Comparing the coefficients of $\frac{t^{n}}{n !}$ on the right hand sides of the last two equations, we arrive at the desired result.

Remark 6. By setting $d=1$ in Theorem 10, we get the following result

$$
\begin{aligned}
& \sum_{m=0}^{n}\left(\begin{array}{c}
n \\
m
\end{array}\right) c^{n-m}{ }_{H} b_{n-m}^{\left(k_{1}, k_{2}, \cdots, k_{r}\right)}(x, y, z)_{H} b_{m}^{\left(k_{1}, k_{2}, \cdots, k_{r}\right)}\left(c x, c y, c^{2} z\right) \\
& =\sum_{m=0}^{n}\left(\begin{array}{c}
n \\
m
\end{array}\right) c^{m}{ }_{H} b_{n-m}^{\left(k_{1}, k_{2}, \cdots, k_{r}\right)}\left(c x, c y, c^{2} z\right)_{H} b_{m}^{\left(k_{1}, k_{2}, \cdots, k_{r}\right)}(x, y, z) .
\end{aligned}
$$

Theorem 11. For all integers $c, d>0$ and $n \geq 0$, the following identity holds true:

$$
\begin{aligned}
\sum_{m=0}^{n} & \left(\begin{array}{c}
n \\
m
\end{array}\right) c^{n-m} d_{H}^{m} b_{n-m}^{\left(k_{1}, k_{2}, \cdots, k_{r}\right)}\left(d x, d y, d^{2} z,\right) \\
& \times \sum_{i=0}^{m}\left(\begin{array}{l}
m \\
i
\end{array}\right) T_{i}(c-1) b_{m-i}^{\left(k_{1}, k_{2}, \cdots, k_{r}\right)}(c w) \\
= & \sum_{m=0}^{n}\left(\begin{array}{l}
n \\
m
\end{array}\right) d^{n-m} c^{m}{ }_{H} b_{n-m}^{\left(k_{1}, k_{2}, \cdots, k_{r}\right)}\left(c x, c y, c^{2} z\right) \\
& \times \sum_{i=0}^{m}\left(\begin{array}{l}
m \\
i
\end{array}\right) T_{i}(d-1) b_{m-i}^{\left(k_{1}, k_{2}, \cdots, k_{r}\right)}(d w) .
\end{aligned}
$$


Proof. Let

$$
\begin{aligned}
h(t)= & \left(\frac{\left(r ! \operatorname{Li}_{k_{1}, k_{2}, \cdots, k_{r}}\left(1-e^{-c t}\right)\right)\left(r ! \operatorname{Li}_{k_{1}, k_{2}, \cdots, k_{r}}\left(1-e^{-d t}\right)\right)}{[(\log (1+c t))]^{r}[(\log (1+d t))]^{r}}\right. \\
& \left.\frac{\left(1-\left(-e^{t}\right)^{c d}\right)}{\left(e^{c t}+1\right)\left(e^{d t}+1\right)}\right)(1+c d t)^{x+w} e^{c d y t+c^{2} c^{2} z t^{2}} \\
h(t)= & \sum_{n=0}^{\infty} H_{n}^{\left(k_{1}, k_{2}, \cdots, k_{r}\right)}\left(d x, d y, d^{2} z\right) \frac{(c t)^{n}}{n !} \\
& \times \sum_{n=0}^{\infty} T_{n}(c-1) \frac{(d t)^{n}}{n !} \sum_{n=0}^{\infty} b_{m}^{\left(k_{1}, k_{2}, \cdots, k_{r}\right)}(c w) \frac{(d t)^{m}}{m !} \\
= & \sum_{n=0}^{\infty} \sum_{m=0}^{n}\left(\begin{array}{c}
n \\
m
\end{array}\right) c^{n-m} d^{m}{ }_{H} b_{n-m}^{\left(k_{1}, k_{2}, \cdots, k_{r}\right)}\left(d x, d y, d^{2} z\right) \\
& \times \sum_{i=0}^{m}\left(\begin{array}{c}
m \\
i
\end{array}\right) T_{i}(c-1) b_{m-i}^{\left(k_{1}, k_{2}, \cdots, k_{r}\right)}(c w) \frac{t^{n}}{n !} .
\end{aligned}
$$

By using the similar method, we can show that

$$
\begin{aligned}
h(t)= & \sum_{n=0}^{\infty} \sum_{m=0}^{n}\left(\begin{array}{c}
n \\
m
\end{array}\right) c^{n-m} d_{H}^{m} b_{n-m}^{\left(k_{1}, k_{2}, \cdots, k_{r}\right)}\left(c x, c y, c^{2} z\right) \\
& \times \sum_{i=0}^{m}\left(\begin{array}{c}
m \\
i
\end{array}\right) T_{i}(d-1) b_{m-i}^{\left(k_{1}, k_{2}, \cdots, k_{r}\right)}(d w) \frac{t^{n}}{n !} .
\end{aligned}
$$

After comparing the coefficients of $\frac{t^{n}}{n !}$ on the right hand sides of the last two equations, we get the result (44).

\section{References}

[1] Andrews L.C., Special functions for engineers and mathematicians, Macmillan. Co., New York, 1985.

[2] Arakawa T., Kaneko M., Multiple zeta values, poly-Bernoulli numbers, and related zeta functions, Nagoya Math. J., 153(1999), 189-209.

[3] Bell E.T., Exponential polynomials, Ann. of Math., 35(1934), 258-277.

[4] Carlitz L., A note on Bernoulli and Euler polynomials of the second kind, Scripta Math., 25(1961), 323-330.

[5] Dattoli G., Lorenzutta S., Cesarano C., Finite sums and generalized forms of Bernoulli polynomials, Rendiconti di Mathematica, 19(1999), 385-391.

[6] Kaneko M., Poly-Bernoulli numbers, J. de Theorie de Nombres, 9(1997), 221-228.

[7] KHAN W.A., A note on Hermite-based poly-Euler and multi poly-Euler polynomials, Palestine J. Math., 5(1)(2016), 17-26. 
[8] Kim T., Kwaon H.I., Lee S.H, Seo J.J., A note on poly-Bernoulli numbers and polynomials of the second kind, Advances in Defference Equations, 219(2014).

[9] Pathan M.A., Khan W.A., Some implicit summation formulas and symmetric identities for the generalized Hermite-Bernoulli polynomials, Mediterr. J. Math., 12(2015), 679-695.

[10] Pathan M.A., Khan W.A., Some new classes of generalized Hermite-based Apostol-Euler and Apostol-Genocchi polynomials, Fasciculi Mathematici, 55(2015), 153-170.

[11] Qi F., Kim D.S., Kim T., Dolgy D.V., Multiple poly-Bernoulli polynomials of the second kind, Advanced Studies in Contemporary Mathematics, 25(2015), $1-7$.

[12] Roman S., The Umbral Calculus, Volume 111 of Pure and Applies Mathematics, Academic Press, Inc, [Harcourt Brace Jovanovich, Publishers], New York, 1984.

[13] Srivastava H.M., Manocha H.L., A treatise on generating functions, Ellis Horwood Limited. Co., New York, 1984.

\author{
WASEem A. KHAN \\ Department of Mathematics \\ FACULTY OF SCIENCE \\ INTEGRAL UNIVERSITY \\ LUCKNOW-226026, INDIA
}

e-mail: waseem08_khan@rediffmail.com

M. GHAYASUDDIN

Department of Mathematics

FACULTY OF SCIENCE

INTEGRAL UNIVERSITY

LUCKNOW-226026, INDIA

e-mail: ghyas.maths@gmail.com

\author{
M. SHADAB \\ Department of Applied Sciences and Humanities \\ FACUlTy OF ENGINEERING AND TECHNOLOGY \\ Jamiamillia Islamia (A Central University) \\ New DelHi-110025, India \\ e-mail: shadabmohd786@gmail.com
}

Received on 31.12.2016 and, in revised form, on 30.04.2017. 